\title{
Global burden of tuberculosis and lower respiratory infections attributable to alcohol consumption in 2004
}

\author{
Kevin D. Shield ${ }^{1,2}$, Andriy V. Samokhvalov ${ }^{1,4}$, and Jürgen Rehm ${ }^{1,2,3,4,5}$ \\ ${ }^{1}$ Centre for Addiction and Mental Health, Toronto, Canada \\ ${ }^{2}$ Institute of Medical Science (IMS), University of Toronto, Toronto, Canada \\ ${ }^{3}$ Institute for Clinical Psychology and Psychotherapy, Technische Universität Dresden, Dresden, Germany \\ ${ }^{4}$ Department of Psychiatry, University of Toronto, Toronto, Canada \\ ${ }^{5}$ Dalla Lana School of Public Health, University of Toronto, Toronto, Canada
}

\begin{abstract}
Aim: To quantify the extent to which alcohol contributes to the global burden of tuberculosis (TB) and lower respiratory infections (LRI).

Design: TB and LRI deaths and disability-adjusted life years (DALYs) lost due to alcohol consumption were calculated from alcohol-attributable fractions (AAFs) using various data sources.

Measures: Deaths and DALYs lost were obtained from the World Health Organization (2004 revision of the Global Burden of Disease study). Alcohol consumption indicators were obtained from the ongoing Comparative Risk Assessment study. Relative risks were obtained from meta-analyses, and confidence intervals (CIs) for the AAFs were obtained using Monte Carlo simulations.

Findings: In 2004 alcohol was responsible for 381,000 deaths (95\% CI: 209,000-560,000), 215,000 from TB (95\% CI: 135,000295,000) and 167,000 from LRI (95\% CI: 74,000-264,000); and 6,101,000 DALYs lost (95\% CI: 3,463,000-8,777,000), 4,581,000 due to TB (95\% CI: 2,835,000 to 6,326,000) and 1,152,000 due to LRI (95\%CI: 954,000-566,000). This represents $0.65 \%$ of all deaths (95\% CI: $0.36 \%-0.95 \%$ ) and $0.40 \%$ of all DALYs lost (95\% CI: $0.23 \%-0.23 \%$ ) for people aged 15 years and older.

Conclusions: The global burden of alcohol-attributable TB and LRI is substantial, and significant attention should be paid to monitoring it. Future research should focus on quantifying alcohol's role in (1) the risk for infection, (2) disease progression, and (3) adherence to medication regimens, in order to ensure accurate descriptions of the resulting global burden attributable to alcohol consumption.
\end{abstract}

Alcohol has a substantial impact on the burden of disease, with infectious diseases suspected to be a major contributor to this burden (Rehm, Anderson et al., 2009; Rehm, Baliunas et al., 2010; World Health Organization [WHO] 2009). However, the role of alcohol in the incidence of infection and its progression, in people's adherence to medication regimens, and in the resulting mortality and morbidity caused by infectious diseases is commonly overlooked (Parry, Rehm, Poznyak, \& Room, 2009), because recent burden of disease and injury estimates provided in the 2004 Comparative Risk Assessment (CRA) study exclude the disability and morbidity caused by infectious disease (WHO, 2009). Inclusion of the burden caused by these diseases that is attributable to alcohol consumption may markedly change the current estimates for this burden, since in 2004 two major infections alone, tuberculosis (TB) and lower respiratory infections (LRI), accounted globally for 5.6 million deaths $(9.4 \%$ of all deaths) and 126.4 million disability-adjusted life years (DALYs) lost (8.3\% of all DALYs lost) (WHO, 2008).

Correspondence: Kevin D. Shield, Centre for Addiction and Mental Health, 33 Russell Street, Toronto, ON, M5S 2S1, Canada

Phone: +1 647971 6175. Fax: +1 416260 4146. E-mail: kevin.shield@utoronto.ca

Competing interests: The authors have declared that no competing interests exist.

Financial support: Financial support for this study was provided to Jürgen Rehm by the U.S. National Institute for Alcohol Abuse and Alcoholism (NIAAA) with contract number HHSN267200700041C to conduct the study titled “Alcohol- and Drug-Attributable Burden of Disease and Injury in the U.S.” Dr. Rehm also received salary and infrastructure support from the Ontario Ministry of Health and Long-Term Care.

Keywords: alcohol, global burden of disease, tuberculosis, lower respiratory infections, deaths, DALYs, infectious diseases 
Though the burden of alcohol-attributable infectious diseases was not included in the most recent CRA study, the impact of alcohol consumption on the incidence of TB and LRI has long been acknowledged (Capps \& Coleman, 1923; MacGregor \& Louria, 1997; Rush, 1943). Research has led to the hypotheses that alcohol consumption and heavy alcohol consumption in particular, contributes to the incidence of infectious diseases and the worsening of the course of the diseases in two major ways: (1) weakening of the immune system (Cook, 1998; MacGregor \& Louria, 1997; Szabo \& Mandrekar, 2009) (for example, in the case of LRI the sedative properties of alcohol lead to a decrease in oropharyngeal tone, increased risk of aspiration, decreased cough reflex, and decreased mucociliary clearance (Nelson \& Kolls, 2002)) and (2) decreasing adherence to medication regimens (Jakubowiak et al., 2008; Nyamathi et al., 2008). In addition, the social drift hypothesis predicts that excessive consumption and/or alcohol use disorders increase the risk of social downstream migration, thereby increasing the likelihood of contagion (Rehm, Samokhvalov et al., 2009). Not only have the biological pathways been clarified, but also the usual epidemiological criteria for causality (Rothman, Greenland, \& Lash, 2008) of the effect of alcohol consumption on TB (Rehm, Samokhvalov et al., 2009) and LRI (Samokhvalov, Irving, \& Rehm, 2010) have been fulfilled.

Although there is a clear understanding of the biological mechanisms of how alcohol influences the probability of infection and disease course for TB and LRI (U.S. Department of Health and Human Services, 2000), research quantifying the alcohol-attributable burden for these two infectious diseases is scarce. Thus, the number of studies needed to obtain stable risk estimates from meta-analyses has not been met until recently (Lönnroth, Williams, Stadlin, Jaramillo, \& Dye, 2008; Rehm, Samokhvalov et al., 2009; Samokhvalov et al., 2010).

Given the lack of information on the alcohol-attributable TB and LRI burden and the recent WHO global strategy to reduce the harmful effects of alcohol (which called for the strengthening of information about alcohol-related harms) (WHO, 2010a), it is the aim of this paper to calculate the alcohol-attributable burden of TB and LRI in terms of deaths and disability-adjusted life years (DALYs) lost, and then to calculate the extent to which this estimated burden would change current estimates of the global burden of disease attributable to alcohol consumption.

\section{Method}

Calculation of alcohol-attributable fractions (AAFs) (defined as the proportion of disease that would not be present if the exposure was eliminated, in this case if every person was a lifetime abstainer from alcohol) involves two steps: (1) calculation of the cause-, sex-, and regionspecific AAFs, and (2) application of these AAFs to mortality and DALYs lost data.

\section{Data for regions}

Global regions were defined according to the 2005 GBD study (Institute for Health Metrics and Evaluation, 2010). Global Burden of Disease (GBD) regions are defined according to geography and are based on child and adult mortality profiles of each country. Population data for regions were obtained from the latest population revisions of the United Nations Population Division (United Nations Population Division, 2010).

\section{Step 1: Calculation of the AAF by sex and age}

This step involves the calculation of the alcoholattributable fractions from data on drinking status, amount of alcohol consumed among current drinkers, and relative risks (RRs), and then the calculation of 95\% CIs for each of the AAFs.

\section{Alcohol consumption measures}

Data on alcohol consumption variables for 2005 were obtained from the 2005 CRA study. For our analysis we used the alcohol consumption indicators of the prevalence of each of current drinkers (defined as those who have had at least one drink in the previous year), former drinkers (defined as those who have not had a drink in the previous year, but who have consumed alcohol within their lifetime), and lifetime abstainers.

Adult per capita consumption estimates (defined as grams of pure alcohol consumed per adult per year) were calculated from recorded consumption, unrecorded consumption and tourist consumption. Estimates of recorded adult per capita alcohol consumption were obtained from the Global Information System on Alcohol and Health database (WHO, 2010b). These estimates were based on government records (taxation), industry publications for the production and sale of alcohol, and data from the U.N. Food and Agriculture Organization (Rehm, Klotsche, \& Patra, 2007). Estimates of unrecorded and tourist adult per capita consumption were taken from the ongoing CRA study within the 2005 GBD study. The main sources for unrecorded consumption were home production, alcohol intended for industrial, technical and medical uses, and illegal production or importation of alcohol (Rehm et al., 2007). As no variance estimates for unrecorded and tourist alcohol consumption existed, we estimated the variance to be five times that of the variance of recorded alcohol consumption proportionate to the mean (Rehm, Kehoe et al., 2010).

Average daily alcohol consumption was calculated based on $80 \%$ of adult per capita consumption, to adjust for the under-coverage that is likely present in the observational studies that were used to calculate pooled relative risks (RRs) in the meta-analyses, and also to account for alcohol that was not consumed.

To describe the distribution of alcohol consumption for the GBD regions, we used a modeling approach suggested by Rehm, Kehoe and colleagues (2010). By using data on average daily alcohol consumption for over 66 countries 
representing 851 distributions, Rehm and colleagues found that alcohol is best modeled in most instances through a gamma distribution. They also found that the mean $(\mu)$ could be used to predict the standard deviation $(\sigma)$ by regressing the means of over 500 average daily alcohol consumption distributions with the standard deviation of the mean. These relationships can be stated as follows:

Formula 1:

$$
\sigma_{\text {men }}=1.171 * \mu_{\text {men }}
$$

Formula 2:

$$
\sigma_{\text {women }}=1.258 * \mu_{\text {women }}
$$

The standard deviation and mean were then used to calculate the shape $(\kappa)$ and scale $(\theta)$ parameters of the gamma distributions and to characterize the alcohol consumption distribution.

\section{Risk relations}

Risk relations for TB were obtained from Lönnroth and colleagues' meta-analysis (Lönnroth, Williams, Stadlin, Jaramillo, \& Dye, 2008) and risk relations for LRI were obtained from Samokhvalov and colleagues' (2010) metaanalysis.

\section{AAF calculations}

AAF calculations were based on the distribution of alcohol consumption, the prevalence of current drinkers, former drinkers, and abstainers, and the risk relations as follows:

$$
A A F=\frac{P_{a b s}+P_{\text {former }} R R_{\text {former }}+\int_{0}^{150} P_{\text {current }}(x) R R_{\text {current }}(x) d x-1}{P_{a b s}+P_{\text {former }} R R_{\text {former }}+\int_{0}^{150} P_{\text {current }}(x) R R_{\text {current }}(x) d x}
$$

where $\mathrm{P}_{a b s}$ is the prevalence of lifetime abstainers, $\mathrm{P}_{\text {former }}$ is the prevalence of former drinkers, $\mathrm{P}_{\text {current }}$ is the prevalence of current drinkers who consume a given average daily alcohol consumption of $x, \mathrm{RR}_{\text {former }}$ is the relative risk for former drinkers, and $\mathrm{RR}_{\text {current }}$ is the relative risk for current drinkers given an alcohol consumption of $x$. In calculating the relative risk of current drinkers we used a cap of 150 grams of alcohol per day and normalized the distribution accordingly, as consumption over this amount is likely to be unsustainable for long periods of time.

\section{$\mathbf{9 5 \%}$ confidence intervals for the $A A F s$}

To calculate the $95 \%$ CIs of the AAFs we used a Monte Carlo-like approach outlined by Gmel and colleagues (2011). To estimate the variance of the AAFs we used 40,000 simulations that estimated the lowest level parameters used in the AAF formulas. For each simulation we generated estimates for the prevalence of "former drinkers" and "lifetime abstainers," from which a prevalence of "current drinkers" was then estimated. Additionally, we generated estimates of the RR function betas. For the $95 \%$ CIs of the average alcohol consumption AAFs, we generated the per capita alcohol consumption and multiplied this by 0.8 . The kappa parameter of the gamma distribution was computed by taking into account the variance between the standard deviation and the mean (Rehm, Kehoe et al., 2010). The gamma parameter was calculated based on the adjusted mean and the kappa parameter. The resulting lowest level parameters were then used to calculate 40,000 AAF estimates, which were then used to calculate the variance of the AAFs and the 95\% CIs for each disease category.

\section{Step 2: Application of the AAFs to region-specific mortality and DALYs lost data}

This step requires multiplying the sex-, region-, and causespecific AAFs by mortality and DALYs lost data respectively.

\section{Outcome data}

To quantify the burden of TB and LRI attributable to alcohol consumption in 2004, we used an event-based measure (mortality) and time-based measures (DALYs lost). Data from 2004 on mortality and DALYs lost for people aged 15 years and older were obtained by country from WHO. The methods used to estimate the mortality and DALYs lost in the GBD project are described elsewhere (Lopez et al., 2006a, 2006b). Estimates of mortality and DALYs lost were available for each country, and were used to calculate regional estimates. This meant that for each region, sex- and cause-specific AAFs were applied to sex- and cause-specific outcome data. Estimates for deaths and DALYs lost for 2004 were standardized per 100,000 people using the world population as the standard population.

All statistics and analyses were performed using $\mathrm{R}$ version 2.11.1.

\section{Results}

Alcohol consumption by GBD region is outlined in Table 1. The regions of North Africa/Middle East, Southeast Asia, and South Asia had the lowest per capita consumption, lowest average daily consumption among drinkers, and lowest prevalence of people who consumed on average more than 40 grams of alcohol per day, while the Eastern European region had the highest values in these categories. In every region, men were more likely to be current drinkers than were women; of those current drinkers men consumed more alcohol daily than did women, and men were more likely than women to be heavy consumers of alcohol (i.e., drinking more than 40 grams of alcohol per day).

Overall, 381,000 TB- and LRI-related deaths (95\% CI: 209,000-560,000) were attributable to alcohol consumption. This number can be broken down into 215,000 deaths (95\% CI: 135,000-295,000) from TB $(180,000$ for men, 35,000 for women), and 167,000 deaths (95\% CI: 74,000264,000) from LRI (95,000 for men, 72,000 for women). Deaths from TB and LRI attributable to alcohol were responsible for $0.65 \%$ (95\% CI: $0.36 \%-0.95 \%$ ) of all deaths ( $0.88 \%$ for men, $0.39 \%$ for women). 
Table 1

Drinking indicators by Global Burden of Disease (GBD) region for 2005

\begin{tabular}{|c|c|c|c|c|c|c|c|c|}
\hline \multirow[b]{2}{*}{ GBD region } & \multicolumn{4}{|c|}{ Men } & \multicolumn{4}{|c|}{ Women } \\
\hline & $\begin{array}{l}\text { Current } \\
\text { drinkers }\end{array}$ & $\begin{array}{l}\text { Former } \\
\text { drinkers }\end{array}$ & $\begin{array}{c}\text { Lifetime } \\
\text { abstainers }\end{array}$ & $\begin{array}{c}\text { Per capita } \\
\text { consumption } \\
\text { (liter/year per } \\
\text { person) }\end{array}$ & $\begin{array}{l}\text { Current } \\
\text { drinkers }\end{array}$ & $\begin{array}{l}\text { Former } \\
\text { drinkers }\end{array}$ & $\begin{array}{c}\text { Lifetime } \\
\text { abstainers }\end{array}$ & $\begin{array}{c}\text { Per capita } \\
\text { consumption } \\
\text { (liter/year per } \\
\text { person) }\end{array}$ \\
\hline $\begin{array}{l}\text { Asia, Pacific } \\
\text { [High Income] }\end{array}$ & $87.43 \%$ & $7.26 \%$ & $5.31 \%$ & 15.23 & $75.62 \%$ & $8.80 \%$ & $15.58 \%$ & 4.63 \\
\hline Asia, Central & $63.87 \%$ & $13.29 \%$ & $22.84 \%$ & 10.62 & $46.83 \%$ & $13.79 \%$ & $39.38 \%$ & 3.23 \\
\hline Asia, East & $71.71 \%$ & $15.36 \%$ & $12.93 \%$ & 9.88 & $37.50 \%$ & $17.56 \%$ & $44.93 \%$ & 1.95 \\
\hline Asia, South & $16.68 \%$ & $10.79 \%$ & $72.53 \%$ & 3.80 & $2.64 \%$ & $7.20 \%$ & $90.17 \%$ & 0.24 \\
\hline Asia, Southeast & $27.21 \%$ & $17.06 \%$ & $55.73 \%$ & 5.21 & $5.63 \%$ & $4.22 \%$ & $90.14 \%$ & 0.47 \\
\hline Australasia & $87.08 \%$ & $6.78 \%$ & $6.14 \%$ & 14.29 & $80.02 \%$ & $8.79 \%$ & $11.19 \%$ & 5.78 \\
\hline Caribbean & $65.17 \%$ & $18.00 \%$ & $16.83 \%$ & 9.36 & $34.23 \%$ & $19.14 \%$ & $46.63 \%$ & 2.74 \\
\hline Europe, Central & $77.41 \%$ & $13.17 \%$ & $9.41 \%$ & 21.81 & $59.05 \%$ & $18.80 \%$ & $22.15 \%$ & 6.70 \\
\hline Europe, Eastern & $71.74 \%$ & $17.28 \%$ & $10.97 \%$ & 25.19 & $50.77 \%$ & $21.23 \%$ & $28.00 \%$ & 8.07 \\
\hline Europe, Western & $87.80 \%$ & $6.47 \%$ & $5.73 \%$ & 17.64 & $77.56 \%$ & $9.38 \%$ & $13.05 \%$ & 7.06 \\
\hline $\begin{array}{l}\text { Latin America, } \\
\text { Andean }\end{array}$ & $67.92 \%$ & $14.53 \%$ & $17.55 \%$ & 11.35 & $47.14 \%$ & $25.54 \%$ & $27.31 \%$ & 3.43 \\
\hline $\begin{array}{l}\text { Latin America, } \\
\text { Central }\end{array}$ & $57.83 \%$ & $15.87 \%$ & $26.30 \%$ & 11.73 & $34.88 \%$ & $17.49 \%$ & $47.63 \%$ & 3.23 \\
\hline $\begin{array}{l}\text { Latin America, } \\
\text { Southern }\end{array}$ & $86.48 \%$ & $6.46 \%$ & $7.06 \%$ & 13.91 & $66.75 \%$ & $22.88 \%$ & $10.37 \%$ & 5.28 \\
\hline $\begin{array}{l}\text { Latin America, } \\
\text { Tropical }\end{array}$ & $58.67 \%$ & $27.00 \%$ & $14.33 \%$ & 14.11 & $41.48 \%$ & $35.64 \%$ & $22.88 \%$ & 4.39 \\
\hline $\begin{array}{l}\text { Northern Africa / } \\
\text { Middle East }\end{array}$ & $8.90 \%$ & $19.29 \%$ & $71.81 \%$ & 2.04 & $2.40 \%$ & $5.74 \%$ & $91.86 \%$ & 0.26 \\
\hline $\begin{array}{l}\text { North America } \\
\text { [High Income] }\end{array}$ & $72.70 \%$ & $15.94 \%$ & $11.36 \%$ & 14.38 & $60.98 \%$ & $17.31 \%$ & $21.71 \%$ & 5.05 \\
\hline Oceania & $79.59 \%$ & $9.07 \%$ & $11.34 \%$ & 5.55 & $47.59 \%$ & $15.61 \%$ & $36.80 \%$ & 0.94 \\
\hline $\begin{array}{l}\text { Sub-Saharan } \\
\text { Africa, Central }\end{array}$ & $49.95 \%$ & $23.35 \%$ & $26.71 \%$ & 5.83 & $29.88 \%$ & $28.03 \%$ & $42.08 \%$ & 2.18 \\
\hline $\begin{array}{l}\text { Sub-Saharan } \\
\text { Africa, East }\end{array}$ & $29.83 \%$ & $14.22 \%$ & $55.95 \%$ & 7.37 & $19.34 \%$ & $11.22 \%$ & $69.44 \%$ & 2.19 \\
\hline $\begin{array}{l}\text { Sub-Saharan } \\
\text { Africa, Southern }\end{array}$ & $37.53 \%$ & $11.21 \%$ & $51.26 \%$ & 14.28 & $13.60 \%$ & $6.61 \%$ & $79.80 \%$ & 3.07 \\
\hline $\begin{array}{l}\text { Sub-Saharan } \\
\text { Africa, Western }\end{array}$ & $41.39 \%$ & $13.65 \%$ & $44.96 \%$ & 11.69 & $24.76 \%$ & $16.41 \%$ & $58.83 \%$ & 3.94 \\
\hline World & $50.22 \%$ & $14.06 \%$ & $35.72 \%$ & 9.74 & $30.82 \%$ & $13.00 \%$ & $56.18 \%$ & 2.60 \\
\hline
\end{tabular}

Figures 1 and 2 outline the number of alcohol-attributable deaths per 100,000 people (standardized based on the age and sex population distribution of the world) for $\mathrm{TB}$ and LRI. Overall, the Sub-Saharan Africa regions had the highest standardized rate of alcohol-attributable deaths from TB, while the Sub-Saharan Africa regions (except the southern Sub-Saharan Africa region) and the Latin American regions had the highest rates of alcoholattributable deaths from LRI.

In $2004,6,101,000 \quad(95 \% \quad$ CI: $\quad 3,463,000-8,777,000)$ DALYs lost (4,737,000 for men, 1,363,000 for women) due to TB and LRI were attributable to alcohol. This represents $0.40 \%$ of all DALYs lost (95\% CI: $0.23 \%-0.23 \%$ ) for 2004 for people 15 years of age and older $(0.59 \%$ for men, $0.19 \%$ for women). This number can be broken down into 4,581,000 DALYs lost (95\% CI: 2,835,000-6,326,000) due to TB (2,522,000 for men, 313,000 for women) and 1,152,000 DALYs lost (95\% CI: 954,000-566,000) due to LRI (954,000 for men, 566,000 for women) attributable to alcohol consumption. Figures 3 and 4 outline the number of alcohol-attributable DALYs lost per 100,000 people (standardized based on the age and sex population distribution of the world) due to TB and LRI. As with overall deaths, the Sub-Saharan Africa regions had the highest standardized rate of DALYs lost due to alcoholattributable TB, while the Sub-Saharan Africa regions (except the southern Sub-Saharan Africa region) and the Latin American regions had the highest rates of DALYs lost due to alcohol-attributable LRI.

\section{Discussion}

In this paper we present the estimated global burden of TB and LRI attributable to alcohol consumption. From our analysis we estimated that the number of deaths attributable to alcohol in 2004 would increase by $14.2 \%$ from 2.26 million to 2.58 million, and the number of DALYs lost 


\section{Figure 1}

Number of tuberculosis deaths attributable to alcohol consumption per 100,000 people by GBD region for 2004, standardized for the global population

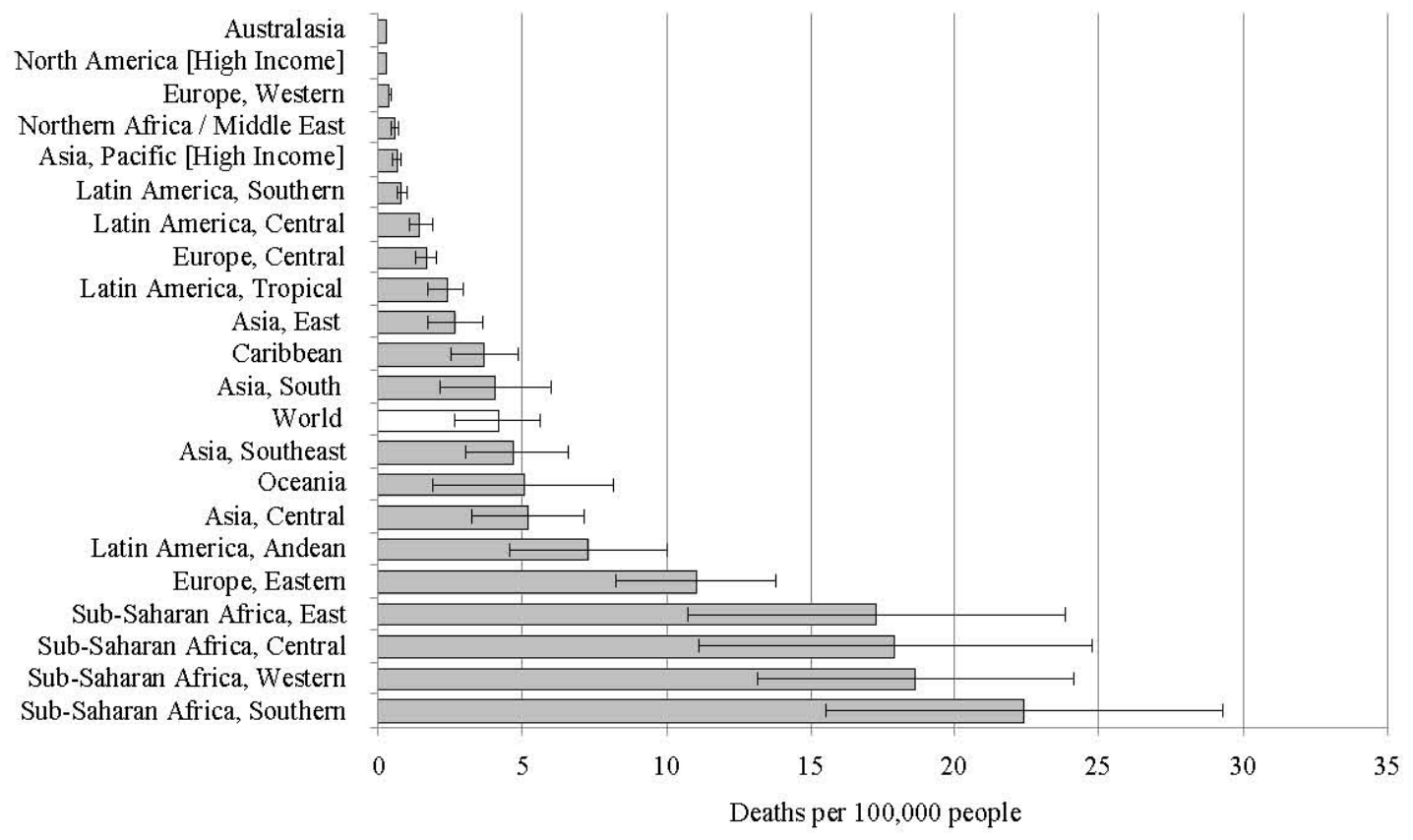

\section{Figure 2}

Number of lower respiratory infection deaths attributable to alcohol consumption per 100,000 people by GBD region for 2004, standardized for the global population

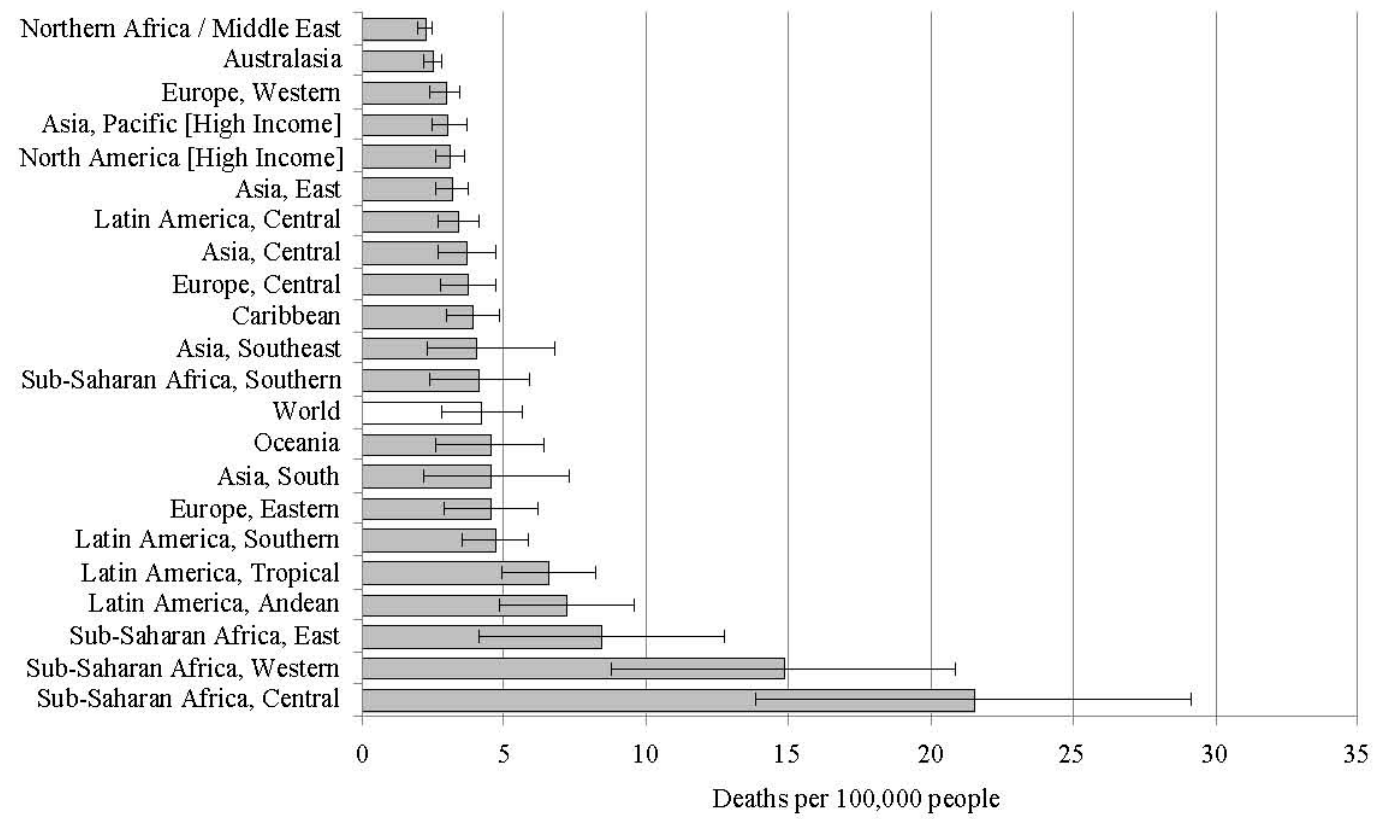




\section{Figure 3}

Number of tuberculosis DALYs lost attributable to alcohol consumption per 100,000 people by GBD region for 2004, standardized for the global population

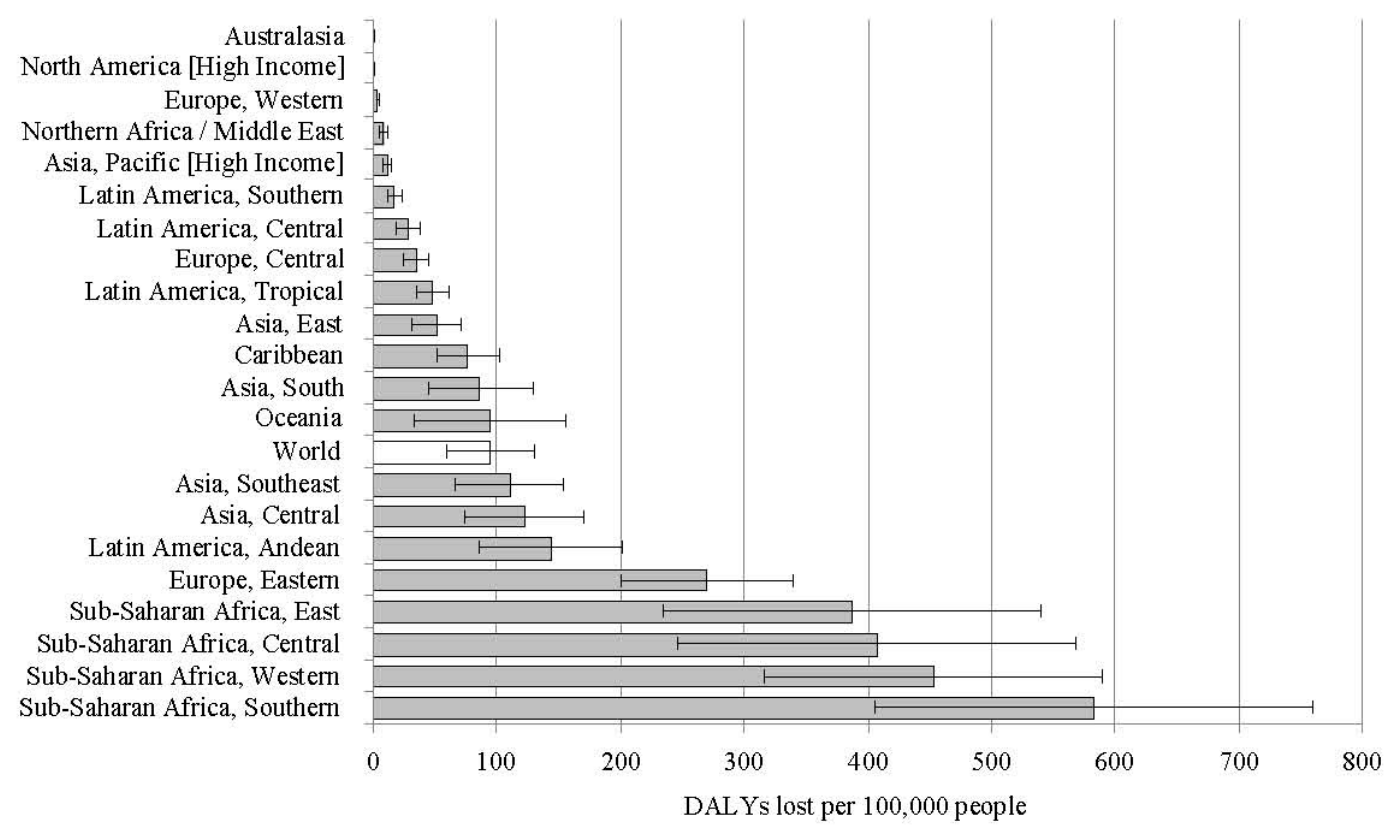

\section{Figure 4}

Number of lower respiratory infection DALYs lost attributable to alcohol consumption per 100,000 people by GBD region for 2004, standardized for the global population

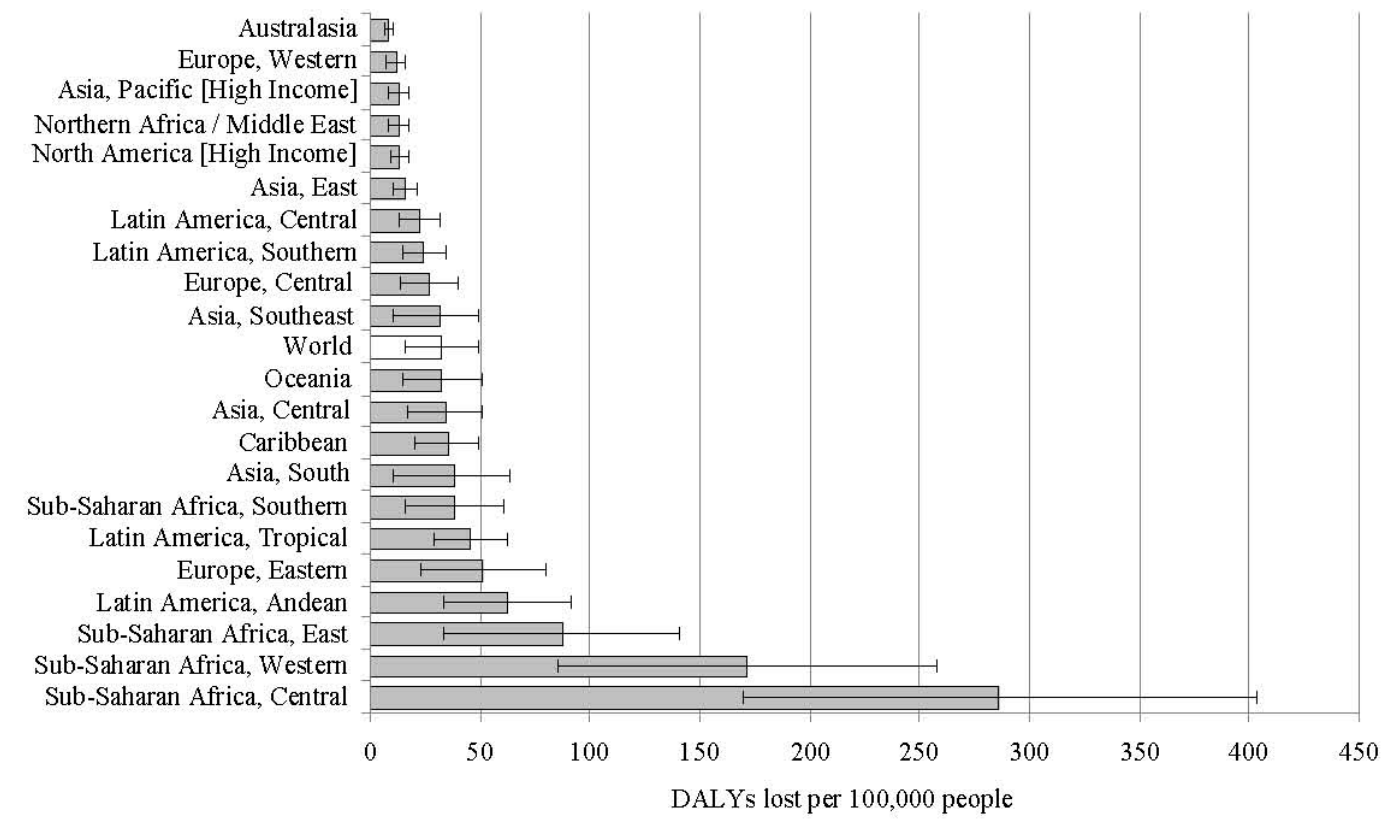


would increase from 70.91 million to 77.01 million, representing an increase of $8.6 \%$ if the deaths and DALYs lost due to TB and LRI due to alcohol consumption were included in the recent estimates outlined by Rehm, Mathers, and colleagues (2009).

Our analysis does have limitations. First, there are data limitations regarding the quality of global mortality data (see Lopez et al., 2006b) which, although not incorporated into our analysis, increase the uncertainty of the number of deaths and DALYs lost. Second, there are weaknesses associated with the assumptions made in the calculation of the DALYs lost (see Rehm \& Frick, 2010), particularly with respect to the calculation of weights for DALYs lost.

Third, the risk relations for community-acquired pneumonia are established, whereas risk relations for the aggregate category of LRI are not (Lönnroth et al., 2008). Since incidence, prevalence, and mortality caused by community-acquired pneumonia are not coded for many regions of the world (WHO, 2008), a global estimate of community-acquired pneumonia does not appear to be obtainable. At the same time, it must be noted that pneumonia and other LRIs, such as bronchitis and lung abscess, share multiple clinical features and many cases coincide or transform into each other, i.e., pneumonia may start as bronchitis. Additionally, pneumonia may transform into abscess where, if the immune system is not able to localize the inflammation and limit the spread of infection, the abscess can spread beyond its clearly demarcated borders and transform into full blown pneumonia. From a pathobiological point of view the biological mechanisms of increased susceptibility to pneumonia and other LRIs are generally the same, and include diminished capacity to eliminate the pathogens (decreased oropharyngeal tone and motility of the cilia of ciliary epithelium) and immunosuppression (Alcón, Fabregas, \& Torres, 2005). Thus, in our analysis, we assumed a homogenous effect of alcohol on all LRI (U.S. Department of Health and Human Services, 2000). Accordingly, we used the risk relationship between alcohol and community-acquired pneumonia as a proxy measurement for all LRIs.

Fourth, alcohol consumption variables used in our analysis came from population surveys that have limitations with respect to coverage. In addition, survey instruments commonly have inherent biases due to self-reporting of data, leading to an underestimation of current drinkers and binge drinkers, and causing an underestimation of the global burden of TB and LRI attributable to alcohol consumption (Shield \& Rehm, 2012).

The recognition, quantification and monitoring of the impact of alcohol consumption on the incidence and progression of infectious diseases is vital, especially in developing countries, where alcohol consumption is increasing and the burden of infectious diseases is greater than in developed countries (WHO, 2008). Given the recent rise of drug-resistant bacteria, the high incidence of LRI and the large global burden of TB and LRI (Centers for Disease Control and Prevention, 1996; WHO, 2008), strategies aimed at reducing alcohol consumption in conjunction with implementing treatment programs for infectious diseases are required in order to reduce the growing global burden of infectious diseases.

\section{References}

Alcón, A., Fabregas, N., \& Torres A. (2005). Pathophysiology of pneumonia. Clinics in Chest Medicine, 1, 39-46.

Capps, J. A., \& Coleman, G. H. (1923). Influence of alcohol on prognosis of pneumonia in Cook County hospital. Journal of the American Medical Association, 80, 750-752.

Centers for Disease Control and Prevention. (1996). Reported tuberculosis in the United States: Surveillance Report 1996. Atlanta, GA, United States: Division of Tuberculosis Prevention, National Center of Infectious Diseases, Centers for Disease Control and Prevention.

Cook, R. T. (1998). Alcohol abuse, alcoholism, and damage to the immune system-A review. Alcoholism: Clinical and Experimental Research, 22, 1927-1942.

Gmel, G. J., Shield, K., Frick, H., Kehoe, T., Gmel, G. S., \& Rehm, J. (2011). Estimating uncertainty for alcoholattributable fractions for infectious and chronic disease. BMC Medical Research Methodology, 11, 48.

Institute for Health Metrics and Evaluation. (2010). Global Burden of Diseases: Injuries and risk factors study operations manual. Seattle, WA, United States: Author.

Jakubowiak, W. M., Bogorodskaya, E. M., Borisov, S. E., Danilova, I. D., Lomakina, O. B., \& Kourbatova, E. V. (2008). Impact of socio-psychological factors on treatment adherence of TB patients in Russia. Tuberculosis, 88, 495-502.

Lönnroth, K., Williams, B., Stadlin, S., Jaramillo E., \& Dye C. (2008). Alcohol use as a risk factor for tuberculosis-A systematic review. BMC Public Health, 8, 289.

Lopez, A. D., Mathers, C. D., Ezzati, M., Jamison, D. T., \& Murray, C. J. L. (2006a). Global and regional burden of disease and risk factors, 2001: Systematic analysis of population health data. Lancet, 367, 1747-1757.

Lopez, A. D., Mathers, C. D., Ezzati, M., Jamison, D. T., \& Murray, C. J. L. (Eds.). (2006b). Global burden of disease and risk factors. New York, NY \& Washington, DC, United States: Oxford University Press and The World Bank.

MacGregor, R. R., \& Louria, D. B. (1997). Alcohol and infection [Review]. Current Clinical Topics in Infectious Diseases, 17, 291-315.

Nelson, S., \& Kolls, J. K. (2002). Alcohol, host defence and society. Nature Reviews Immunology, 2, 205-209.

Nyamathi, A., Nahid, P., Berg, J., Burrage, J., Christiani, A., Aqtash, S., . . Leake B. (2008). Efficacy of nurse case-managed intervention for latent tuberculosis among homeless subsamples. Nursing Research, 57, 33-39. 
Parry, C. D. H., Rehm, J. R., Poznyak, V., \& Room, R. (2009). Alcohol and infectious diseases: Are there causal linkages? Addiction, 104, 331-332.

Rehm, J., Anderson, P., Kanteres, F., Parry, C. D., Samokhvalov, A. V., \& Patra, J. (2009). Alcohol, social development and infectious disease. Stockholm, Sweden: Ministry of Health and Social Offices. Retrieved from http://www.se2009.eu/polopoly_fs/ 1.21749\%21menu/standard/file/ALCOHOL1.PDF

Rehm, J., Baliunas, D., Borges, G. L. G., Graham, K., Irving, H. M., Kehoe, T., .. . Taylor. B. (2010). The relation between different dimensions of alcohol consumption and burden of disease-An overview. Addiction, 105, 817-843.

Rehm, J., \& Frick, U. (2010). Valuation of health states in the U.S. study to establish disability weights: Lessons from the literature. International Journal of Methods in Psychiatric Research, 19, 18-33.

Rehm, J., Kehoe, T., Gmel, G., Stinson, F., Grant, B., \& Gmel, G. (2010). Statistical modeling of volume of alcohol exposure for epidemiological studies of population health: The US example. Population Health Metrics, 8, 3.

Rehm, J., Klotsche, J., \& Patra, J. (2007). Comparative quantification of alcohol exposure as risk factor for global burden of disease. International Journal of Methods in Psychiatric Research, 16, 66-76.

Rehm, J., Mathers, C., Popova, S., Thavorncharoensap, M., Teerawattananon, Y., \& Patra, J. (2009). Global burden of disease and injury and economic cost attributable to alcohol use and alcohol use disorders. Lancet, 373, 2223-2233.

Rehm, J., Samokhvalov, A. V., Neuman, M. G., Room, R., Parry, C. D., Lönnroth, K., . . . Popova, S. (2009). The association between alcohol use, alcohol use disorders and tuberculosis (TB). A systematic review. BMC Public Health, 9, 450.

Rothman, K. J., Greenland, S., \& Lash, T. L. (2008). Modern Epidemiology (3rd ed.). Philadelphia, PA, United States: Lippincott Williams \& Wilkins.
Rush, B. (1943). An enquiry into the effects of ardent spirits upon the human body and mind with an account of the means of preventing and of the remedies for curing them. Boston, MA, United States: James Loring.

Samokhvalov, A. V., Irving, H. M., \& Rehm, J. (2010). Alcohol consumption as a risk factor for pneumonia: Systematic review and meta-analysis. Epidemiology and Infection, 138, 1789-1795.

Shield, K., \& Rehm, J. (2012). Difficulties with telephonebased surveys on alcohol in high-income countries: The Canadian example. International Journal of Methods in Psychiatric Research, 21, 17-28.

Szabo, G., \& Mandrekar, P. (2009). A recent perspective on alcohol, immunity, and host defense. Alcoholism: Clinical and Experimental Research, 33, 220-232.

U.S. Department of Health and Human Services. (2000). 10th special report to the U.S. Congress on alcohol and health. Bethesda, MD, United States: Author. Retrieved from NIAAA.pubs.niaaa.nih.gov/ publications/10report/intro.pdf.

United Nations Population Division. (2010). World populations prospects: The 2008 revision. New York, NY, United States: United Nations.

World Health Organization. (2008). The global burden of disease: 2004 update. Geneva, Switzerland: Author.

World Health Organization. (2009). Global health risks: Mortality and burden of disease attributable to selected major risks. Geneva, Switzerland: Author.

World Health Organization. (2010a). Global strategy to reduce the harmful use of alcohol. Geneva, Switzerland: Author. Retrieved from http://www.who. int/substance_abuse/activities/globalstrategy/en/index. html

World Health Organization. (2010b). Global Information System on Alcohol and Health. Geneva, Switzerland: Author. Retrieved from http://apps.who.int/globalatlas /default.asp 